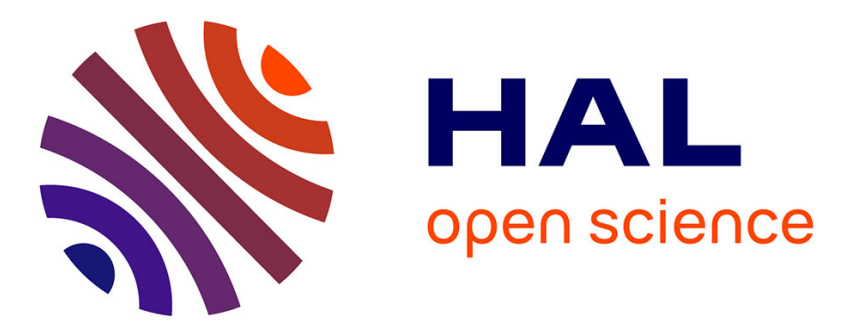

\title{
L'identité géographique de l'Europe: Mer et Montagne dans la Nouvelle Géographie universelle (1876-1894) d'Élisée Reclus
}

Ferretti Federico

\section{- To cite this version:}

Ferretti Federico. L'identité géographique de l'Europe: Mer et Montagne dans la Nouvelle Géographie universelle (1876-1894) d'Élisée Reclus. Alain Cabantous, Jean-Luc Chappey, Renaud Morieux, Nathalie Richard, François Walter. Mer et Montagne dans la culture européenne (XVIe - XIXe siècle), Presses Universitaires de Rennes, pp.175-188, 2011. hal-00662346

\section{HAL Id: hal-00662346 https://hal.science/hal-00662346}

Submitted on 23 Jan 2012

HAL is a multi-disciplinary open access archive for the deposit and dissemination of scientific research documents, whether they are published or not. The documents may come from teaching and research institutions in France or abroad, or from public or private research centers.
L'archive ouverte pluridisciplinaire HAL, est destinée au dépôt et à la diffusion de documents scientifiques de niveau recherche, publiés ou non, émanant des établissements d'enseignement et de recherche français ou étrangers, des laboratoires publics ou privés. 


\section{Federico Ferretti}

Universités de Bologne et Paris I Panthéon - Sorbonne, UMR 8504 Géographie-Cités, équipe E.H.GO - Épistémologie et Histoire de la Géographie

\section{L'identité géographique de l'Europe : mer et montagne dans la Nouvelle géographie universelle (1876-1894) d'Élisée Reclus}

«Or, les montagnes sont plus difficiles à connaître que les cours d'eau, sans doute parce que les fleuves ont un tracé linéaire alors que les montagnes ont une certaine épaisseur ». BROC N., Les montagnes au siècle des Lumières, Paris, CTHS, 1991, p. 57.

\section{Mer et montagne : envisager l'Europe}

Les 19 tomes de la Nouvelle géographie universelle [dorénavant NGU] ${ }^{1}$ d'Élisée Reclus (1830-1905) ont un rôle méconnu mais important dans l'histoire de la géographie et dans l'histoire de la culture européenne, parce qu'ils sont le point d'arrivée du parcours dans lequel la science géographique se développe pendant le $\mathrm{XIX}^{\mathrm{e}}$ siècle $^{2}$, après les ouvrages de Carl Ritter, Alexandre de Humboldt et les Précis de Conrad Malte-Brun ${ }^{3}$. Dans ses écrits théoriques Carl Ritter (1779-1859), dont Reclus a été élève à l'Université de Berlin, recommandait aux géographes l'étude des continents sur la surface du globe selon la dimension horizontale, dite géographique, autant que d'après celle verticale, dite physique. En comparant telle charpente au développement historique des peuples des différentes parties du globe, on peut envisager ses rapports avec l'histoire de l'humanité. La science géographique essaie donc à cette époque d'établir des principes généraux de corrélation entre des faits physiques et des faits humains.

Reclus, qui consacre à l'Europe les cinq premiers volumes de son ouvrage majeur, assigne tant à la mer qu'à la montagne des rôles constitutifs de l'objet Europe. Il s'agit de deux aspects de la dimension physique, qui par son épaisseur pose des problèmes de représentation à la cartographie traditionnelle. La montagne est une barrière, presque la seule limite naturelle dont le géographe arrive à reconnaître l'existence : il nie cette fonction, par exemple, aux fleuves. Mais l'existence d'une telle limite ne légitime pas les frontières politiques qu'y passent : Reclus représente la montagne en tant qu'abri de minorités et de

\footnotetext{
${ }^{1}$ RECLUS É., Nouvelle géographie universelle [dorénavant NGU], Paris, Hachette, 1876-1894.

${ }^{2}$ Sur la continuation du genre de la géographie universelle au siècle suivant voir : RoBIC M.-C. (dir.), Couvrir le monde, un grand $X X^{e}$ siècle de géographie française, Paris, ADPF, 2006.

${ }^{3}$ Malte-Brun C., Précis de géographie universelle, Paris, F. Buisson, 1810-1829.
} 
peuples qui là, où le pouvoir politique est le plus faible, ont pu garder leur liberté. La mer, au contraire, depuis la Méditerranée ancienne jusqu'aux transports transocéaniques de cette époque, a toujours été le véhicule de la civilisation, des voyages, des communications.

Reclus n'emploie pas les montagnes en tant que limites de l'Europe. La ligne conventionnelle de l'Oural ne constitue, à son avis, ni une frontière ethnographique, ni une véritable frontière naturelle, à cause de la faible élévation de telles montagnes. On pourrait dire, avec Ritter, « eine historische Grenze zwischen den Völkern und Reichen Asiens und

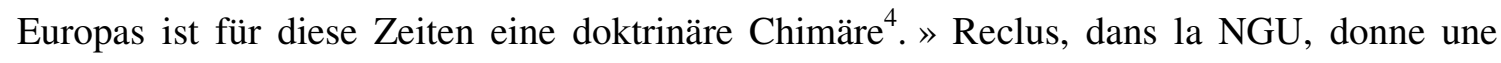
définition des limites orientales de l'Europe qui correspond à la bande de la dépression aralocaspienne qui se trouve au-dessous du niveau de la mer, à l'est de l'Oural. Elle va de la mer Arctique près de l'embouchure de l'Ob jusqu'à l'ancienne limite proposée par Strabon, qui passait «par les palus Méotide et le cours du Tanaïs ${ }^{5}$, » c'est-à-dire la mer d'Azov et le cours du Don. Cette dépression, d'après Reclus, correspond à peu près à l'ancienne mer Hyperboréenne, dont l'existence commençait à être reconnue d'après des preuves géologiques.

Si mer et montagne donnent à l'Europe, dans le discours reclusien, un premier encadrement à l'échelle du continent, il faut voir comment ils agissent à l'échelle des régions. On essayera de cerner les concepts principaux que Reclus emploie et les éléments d'originalité de cet intellectuel politiquement hétérodoxe face à la culture de son époque.

\section{L'Épaisseur de l'Europe : la montagne}

\section{Les montagnes dans l'histoire de la géographie}

La conquête scientifique de la montagne européenne a été longue et difficile pour les géographes. L'idée des anciens Romains qu'il y avait un ager familier dans les plaines à côté d'un saltus dangereux, barbare et inconnu sur les cimes, a duré jusqu'au XVII siècle, qui «ne voyait dans les montagnes que désordre et $\operatorname{chaos}^{6}$. » Dans le cadre de la découverte scientifique des montagnes au siècle suivant, Philippe Buache (1700-1783) a envisagé le premier le concept de bassin hydrographique, en l'encadrant dans une théorie générale des montagnes. «Du tracé des cours d'eau, on pourra déduire la disposition des principales chaînes de montagnes. » Ainsi pour Buache, « la fonction principale des chaînes de montagne est de séparer les bassins fluviaux, qui forment alors à la surface de la terre autant d'unités

\footnotetext{
${ }^{4}$ RitTER C., Europa, Berlin, Georg Reimer, 1863, p. 55.

${ }^{5}$ ReClus É., NGU, vol. I, l'Europe Méridionale, Paris, Hachette, 1876, p. 10.

${ }^{6}$ BROC N., Les montagnes au siècle des Lumières, Paris, CTHS, 1991, p. 47.
} 
naturelles ${ }^{7}$. » Voilà le point qu'on considère le plus faible de cette théorie, parce que cela amènera nombre de cartographes à inventer des chaînes de montagnes là où il n’y avait rien, en les déduisant de tel ou tel bassin fluvial.

Ritter a critiqué le premier, parmi les géographes, cette théorie, en affirmant que le ruissellement peut modifier une chaîne de montagnes, mais qu'il ne peut pas être son origine : l'hydrographie n'explique pas l'orographie. Ritter propose des nombreux exemples empiriques de lieux où il y a des lignes de faîte, mais où l'on ne trouve aucune montagne. Même dans les montagnes les plus connues de l'Europe, les Alpes et les Pyrénées, il y a des lignes de partage des eaux qui ne correspondent pas à l'orientation des chaînes principales. Il refuse donc de procéder de façon «arbitraire, autrement dit en fondant son jugement davantage sur les cartes que sur l'observation de la nature ${ }^{8}$. »

La première règle méthodologique, d'après Ritter, est de procéder d'observation en observation pour en tirer une classification des types de montagne qui manque à son époque. «Ce n'est que lorsque l'individualité des divers systèmes de montagnes sera mieux connue que l'on pourra seulement élaborer une terminologie plus exacte de leurs formes typiques, qui sont hautement variées, et de leurs rapports, dont la géographie ne tient souvent encore compte $^{9}$. » Tel procédé permet de cerner le monde dans toute son épaisseur : ce que Ritter, et ensuite Reclus, reprochent à la carte géographique est surtout de ne pas contenir la troisième dimension du monde.

\section{La montagne de Reclus : une démarche politique}

Reclus s'occupe de l'étude de la montagne depuis les débuts de sa carrière de géographe : dans les années 1860 il travaille à la rédaction de la série Hachette de guides «Joanne » pour lesquels il accomplit plusieurs escalades et voyages d'étude dans les Alpes et les Pyrénées. Son originalité commence à apparaître dans un livre qui peut être considéré, avec son jumeau Histoire d'un ruisseau, comme une introduction à la $\mathrm{NGU}^{10}$ : 1'Histoire d'une montagne. La conquête scientifique des montagnes y est une métaphore de l'affranchissement de l'humanité de la terreur divine et des superstitions religieuses. La science va chasser progressivement les dieux qui les habitaient jadis : «Les dieux s'en vont, emmenant avec eux le cortège des rois, leurs tristes représentants sur la terre [...] Leurs

\footnotetext{
${ }^{7}$ Broc N., La géographie des philosophes : géographes et voyageurs français au XVIII ${ }^{e}$ siècle, Paris, Éditions Ophrys, 1974, p. 202.

${ }^{8}$ RITTER C., Introduction à la géographie générale comparée, Paris, Les Belles Lettres, 1974, p. 89.

${ }^{9}$ RitTER C., Géographie générale comparée, Bruxelles, Établissement Encyclographique, 1837, p. 91.

${ }^{10}$ RAFFESTIN C., «Storia di un ruscello », SCHMIDT DI FRIEDBERG M. (dir.) Élisée Reclus : natura e educazione, Milano, Bruno Mondadori, 2007, p. 294-296.
} 
tonnerres et leurs avalanches ont cessé d'être pour nous les foudres de Jupiter ; leurs nuages ne sont plus les robes de Junon. Sans peur désormais, nous abordons les hautes vallées, résidence des dieux ou repaire des génies ${ }^{11}$. » Bel exemple de la confiance en la science rationnelle de la part d'un géographe qui est aussi évolutionniste et militant anarchiste. ${ }^{12}$

Pour ce qui concerne la structure de l'Europe, les montagnes sont l'expression de ses subdivisions internes les plus importantes. Le système montagneux qui de l'est à l'ouest commence par les Carpates, se poursuit avec les Alpes et se connecte idéalement, à travers le Massif Central français, à la chaîne pyrénéenne, constitue d'après Reclus une ligne unique taillant en deux l'Europe. Cela n'est pas une simple considération morphologique, car chez ce géographe les choix de découpage territorial correspondent toujours à une démarche humaine et historique : «L'épaisseur des Alpes et de tous ses avant-monts, du Pinde aux Carpates, séparait donc vraiment deux mondes distincts où la marche de l'histoire devait s'accomplir différemment ${ }^{13}$.» On envisage d'une côté une Europe méditerranéenne, ouverte aux migrations des peuplades qui portèrent la civilisation la plus ancienne et la répandirent à travers les eaux de la mer qui sera le berceau de la puissance des cités grecques et de Rome ensuite. De l'autre côté, l'Europe continentale et océanique est plus isolée face à de tels mouvements, au moins jusqu'au Moyen Age. En se fondant sur la métaphore ritterienne des continents en tant qu'individus géographiques, Reclus présente le système des montagnes européennes comme la colonne vertébrale de l'individu Europe. Il lui a donné la solidité et la force pour se défendre des attaques de ses redoutables voisins asiatiques tout en gardant sa liberté et en favorisant la survivance de nombreuses enclaves et particularités locales.

«La forme des massifs alpins et du labyrinthe des chaînes orientales devait exercer sur l'histoire de l'Europe, et par conséquent du monde entier, l'influence la plus décisive. Les seules routes des Barbares étant celles qu'avait ouvertes la nature, les peuples asiatiques ne pouvaient pénétrer en Europe que par deux voies, celle de la mer ou celle des grandes plaines du Nord. [...] Les populations nomades et conquérantes qui venaient se heurter contre cet obstacle risquaient d'y briser leur force ${ }^{14}$. »

L'autre aspect caractéristique de la montagne européenne est l'homme qui l'habite, dont la société, selon Reclus, se distingue par la relative liberté dont jouissent les

\footnotetext{
${ }^{11}$ Reclus É., Histoire d'une montagne, Paris, Hetzel, 1880, p. 237.

${ }^{12}$ Voir: Pelletier PH., Élisée Reclus, géographie et anarchie, Paris, Éditions du Monde Libertaire, 2009.

${ }^{13}$ ReCLus É., NGU, vol. I, op. cit., p. 16.

${ }^{14}$ Ibid., p. 15.
} 
communautés montagnardes. La première définition donnée par un géographe des peuples de la montagne remonte à Strabon. Elle se ressent du jugement négatif que son époque donnait du saltus : il accusait ses habitants d'être des pillards, car leur milieu ne leur permettait pas de se livrer à une économie agricole. «Au commencement de cette anomie furent les montagnards, ce qui est normal : ceux-ci en fait, en cultivant des terres peu fertiles et en obtenant des faibles produits, commencèrent à désirer les biens des autres ${ }^{15}$. $\gg$ En dehors de la géographie, l'époque moderne établit, avec des auteurs tels que Bodin et Montesquieu, une caractérisation des peuples montagnards ayant moins de finesse de ceux de la plaine.

Chez Reclus, la montagne se présente comme abri dès les premières pages de l'Histoire d'une montagne, où le narrateur voyage vers l'asile des hautes cimes, déçu de sa vie précédente. «J'avais quitté la région des grandes villes, des fumées et du bruit ; derrière moi étaient restés ennemis et faux amis. Pour la première fois depuis longtemps, j’éprouvai un mouvement de joie réelle. Mon pas devint plus allègre, mon regard plus assuré. Je m'arrêtai pour aspirer avec volupté l'air pur descendu de la montagne ${ }^{16}$. » Il s'agit là d'un lieu commun de la littérature romantique, présent aussi chez Michelet, mais Reclus insère ces considérations dans le cadre de son idée fédéraliste et libertaire. Le géographe lui-même est réfugié dans les montagnes suisses après sa participation à la Commune de Paris ${ }^{17}$.

\section{Montagnes et régions d'Europe dans la NGU}

Nous procéderons pour la montagne, ainsi que pour la mer, d'après la même succession des régions d'Europe qu'on retrouve dans les premiers cinq volumes de la NGU, partant des presqu'iles méditerranéennes, siège de l'histoire la plus ancienne du continent, pour arriver à l'Europe Nord-Occidentale, protagoniste de son expansion contemporaine.

L'idée de liberté montagnarde se lie d'abord aux luttes de libération nationale en cours dans la presqu'île balkanique. Reclus compare les Serbes de la plaine du Danube, qui en 1876 étaient encore en lutte pour leur indépendance, avec ceux de la Montagne Noire, c'est-à-dire les seuls jamais asservis : «Les Monténégrins n’ont jamais été asservis par les Turcs. Tandis que tout le reste du grand empire serbe était envahi par les Osmanlis, eux seuls, grâce à la citadelle de montagnes dans laquelle ils avaient cherché refuge, ont pu maintenir leur indépendance $^{18}$. »

\footnotetext{
${ }^{15}$ STRABON, Géographie, III, 3, 5.

${ }^{16}$ RECLUS É., Histoire d'une montagne, op. cit., p. 3.

${ }^{17}$ Voir : DunBar G., Élisée Reclus historian of nature, Hamden, Archon Books, 1978, p. 58-68 ; FERRETTI F., Il mondo senza la mappa, Élisée Reclus e i geografi anarchici, Milano, Zero in Condotta, 2007, p. 71-74.

${ }^{18}$ RECLUS É., NGU, vol. I, op. cit., p. 293.
} 
En abordant l'autre côté de l'Europe méditerranéenne, le géographe analyse avec intérêt d'autres peuples montagnards, retranchés dans l'aire pyrénéenne et cantabrique de la Péninsule Ibérique, qui avaient résisté à plusieurs invasions en gardant leur langue et leur identité. On est alors à la fin de l'expérience républicaine espagnole de 1868-1874 (Sexenio Democrático), soutenue politiquement par Reclus. Ce n'est pas par hasard qu'il rappelle que dans les massifs asturiens on garde encore le souvenir du mythique royaume de Sobrarbe et que plusieurs populations des Pyrénées, du «pays républicain » du val d'Andorre jusqu'aux Pays Basques, devaient leur indépendance relative à leur pays « si favorable aux partisans ${ }^{19}$. »

Ces peuples ont gardé un grand respect pour la liberté de l'individu : l'exercice de la justice par les assemblées de village, d'après Reclus, en est un exemple. Il analyse l'institution des fors, ou fueros, répandue en diverses contrées de l'aire pyrénéenne, mais appliquée par les Basques avec une singulière rigidité. «Ces fueros, ou droits particuliers des Basques, sont censés les mêmes qu'en l'année 1332, époque à laquelle les députés des provinces se présentèrent à Burgos pour offrir le titre de "seigneur" au roi de Castille. En vertu du traité qui fut conclu, il est interdit au souverain étranger de posséder aucune forteresse, aucun village, aucune maison sur le territoire euskarien ${ }^{20}$. » Reclus, qui apprécie ces limitations du pouvoir royal, critique néanmoins l'interprétation conservatrice qu'en donnent les Basques à son époque, en ironisant sur l'insuffisante diffusion chez eux de l'instruction primaire, car les fueros médiévaux ne la prévoyaient pas!

De l'autre côté des Pyrénées, en France, les conditions de l'orographie font de telle région «le groupe des provinces le plus indépendant ${ }^{21}$.» Son cadre ethnique est composé de peuplades catalanes et basques jalouses de leurs langues et de leurs mœurs autant que les montagnards de la côté ibérique. Ce n'est que l'un des nombreux exemples où Reclus questionne l'unité de la France en soulignant plusieurs particularités historiques et ethniques, qui rendent ces peuples pyrénéens peu portés à la soumission à l'État Français. «Les jeunes hommes, abhorrant pour la plupart le service militaire [...] ne craignent pas d'expatrier et de s'enfuir en Amérique ${ }^{22}$. »

Si les Pyrénées, sont encore pour Reclus une véritable barrière entre deux nations, on trouve dans les Alpes des exemples de son intérêt pour tous les moyens de communication qui mettent les différents peuples en relation mutuelle. La possibilité de connecter Turin à la ligne ferroviaire entre Paris, Lyon et Marseille, mènera selon le géographe à la suppression des

\footnotetext{
${ }^{19}$ Ibid., p. 850.

${ }^{20}$ Ibid., p. 863.

${ }^{21}$ ReClus É., NGU, vol. II, la France., Paris, Hachette, 1877, p. 55.

${ }^{22}$ Ibid., p. 87.
} 
frontières : il affirme que si le Mont Blanc est « encore l'une des principales bornes politiques $\mathrm{d}^{\prime}$ Europe» ${ }^{23}$, bientôt il ne le sera plus.

Dans le troisième volume Reclus aborde le pays qui lui avait donné asile, la Suisse, où il envisage des autres formes de la liberté montagnarde. «C'est en grand partie à la nature que les Suisses sont redevables de leurs libertés politiques et du maintien de leur indépendance nationale : les montagnes, les lacs, les vallées tortueuses ont fait autant que leur vaillance et que la force de leur bras pour les mettre au premier rang parmi les peuples libres ${ }^{24}$. » On voit ici un autre exemple de l'emploi par le géographe d'un langage très proche de celui de l'actualité politique du moment. Il regarde aussi avec intérêt la survivance d'anciennes mœurs communautaires. «Les pâturages des montagnes sont encore, soit des allmends, c'est-à-dire des propriétés communes d'une ville ou d'un village, soit des domaines appartenant à des associés, et de même que dans le Jura français, le beurre et le fromage y sont préparés à frais communs $^{25}$. »

Mais Reclus envisage aussi le problème social de la montagne européenne: l'émigration, notamment vers l'étranger, car les difficiles conditions de vie des hautes montagnes poussent beaucoup de jeunes gens à se déplacer. Le géographe aborde également les problèmes du goitre et du crétinisme. Les hygiénistes contemporains incriminent les conditions hygiéniques et environnementales. Reclus connecte le phénomène à la pauvreté et propose de le vaincre par l'amélioration du niveau de vie et par l'éducation. Bien que scientifiquement très naïve, cette proposition est un exemple de la constante problématique du réformateur social qui s'exprime souvent sous la plume du géographe. A propos d'hygiène et de santé, à l'époque de Reclus la Suisse est à l'avant-garde de l'industrie naissante du tourisme et des vacances. Le géographe cite Rousseau, qui s'étonnait du fait que les bains d'air pur et bienfaisant des montagnes ne fussent prescrits par les textes classiques de la médecine ${ }^{26}$.

En abordant la partie orientale des Alpes, Reclus souligne d'abord qu'en cet endroit la chaîne alpine a exercé plus directement sa fonction historique de défense de l'Europe. «Le système des Alpes orientales forme avec la Suisse, qui jadis fut elle-même autrichienne, un ensemble géographique d'une puissante unité ; c'est la grande forteresse d'Europe ${ }^{27}$. » Plus à l'est encore les Carpates, l'un des derniers massifs explorés scientifiquement, sont considérés

\footnotetext{
${ }^{23}$ Ibid., p. 211.

${ }_{25}^{24}$ Reclus É., NGU, vol. III, l'Europe Centrale, Paris, Hachette, 1878, p. 84.

${ }^{25}$ Ibid., p. 110.

${ }^{26}$ Ibid., p. 67-68.

${ }^{27}$ Ibid., p. 136.
} 
par Reclus comme «le promontoire extrême de la véritable Europe dans les plaines à demi asiatiques de l'Orient Sarmate. C'est contre ce rempart semi-circulaire que tant de peuples, pareils aux flots qui viennent frapper le taille-mer d'un navire, sont venus se heurter sans trouver un passage ${ }^{28}$. $\gg$ La lutte entre l'Europe et l'Asie est donc aussi la lutte entre ces systèmes montagneux et les grandes plaines de l'est. Le meilleur soldat de l'armée européenne, d'après une autre métaphore reclusienne, a été le relief du sol.

Tous les autres complexes orographiques européens ne peuvent être que des objets secondaires face au rôle historique du système principal. Cependant, leurs peuples sont comparés, par genre de vie, économie et esprit d'indépendance, à l'humanité qu'on a vu sur toutes les montagnes du continent : «Les montagnards de l'Écosse étaient encore récemment, comme les Monténégrins, les Mirdites, les Albanais, des peuplades de pasteurs guerriers, toujours en lutte avec leurs voisins : c'est à grand peine qu'on a pu les réduire par la construction de forts au confluent des vallées ${ }^{29}$. »

La montagne européenne est ainsi chez Reclus un objet d'étude complexe, avec des caractéristiques qui peuvent changer à l'échelle de l'histoire. Elle est toujours un abri, un moyen de défense pour les minorités et les plus faibles; elle peut constituer une frontière, mais seulement où elle n'a pas encore été rendue perméable par le travail humain. On la peut considérer comme une limite dynamique qui favorise quelquefois la coexistence de plusieurs ethnies au lieu de les séparer.

\section{L'Épaisseur de l'Europe : la mer}

\section{Méditerranée et civilisation}

A propos de la mer on trouve dans les conclusions de la NGU un bilan des derniers progrès faits par la science dans l'étude de la dimension physique, non seulement en direction des hauteurs, mais aussi des profondeurs : «On a même commencé d'explorer avec méthode le monde souterrain, des cavernes, des catavothres de la Grèce, aux avens et aux puits de Vaucluse et des Causses. La carte des profondeurs marines, avec leur température, leurs organismes vivants, leurs dépôts géologiques, se poursuit et se complète comme celle des continents $^{30}$. » La géographie est donc arrivée à trouver 1 ' « endroit du corail ${ }^{31} »:$ telle était la tâche de Luigi Ferdinando Marsigli, l'un des premiers qui avaient essayé d'élaborer une «théorie générale de la Terre » et de ses eaux, lorsqu'on doutait encore, au début du XVIIIe

\footnotetext{
${ }^{28}$ Ibid., p. 301.

${ }^{29}$ Reclus É., NGU, vol. IV, l'Europe du Nord-Ouest, Paris, Hachette, 1879, p. 723.

${ }^{30}$ Reclus É., NGU, vol. XIX, Amérique du Sud : l'Amazonie et la Plata, Paris, Hachette, 1894, p. 794.

${ }^{31}$ FARINELLI F., I segni del mondo, Firenze, La Nuova Italia, 1992, p. 84.
} 
siècle, que les abîmes marins eussent un fond mesurable. C'est notamment en observant les pêcheurs de corail que Marsigli avait eu l'idée d'employer une sonde pour mesurer scientifiquement les profondeurs du golfe du Lion.

D'après Reclus l'Europe se caractérise parmi tous les continents par sa riche l'articulation littorale ${ }^{32}$ qui a favorisé l'essor de l'économie et de la culture, notamment sur le rivage de la Méditerranée. Reclus définit les îles et promontoires de la Grèce ancienne comme «ces replis de cerveau où s'élabore la pensée de l'homme ${ }^{33}$ ». On sait combien la NGU a contribué à inventer la Méditerranée en tant qu'objet géographique et que «personnage historique $^{34}$.»

Reste à comprendre quel est, selon l'auteur de la NGU, le rôle de la Méditerranée à son époque et quelle est la relation du continent avec ses autres mers. Si à propos de la montagne Reclus cite souvent une littérature très classique et assez connue à l'époque romantique, il emploie à propos de la mer des sources plus récentes, en abordant les derniers progrès de la navigation et de la pêche.

Un phénomène qui concerne les eaux de la Méditerranée, plus salées et plus profondes que celles océaniques, « est la rareté de la vie animale ${ }^{35}$. » Ce que l'homme a pu tirer de cette mer au point de vue alimentaire n'est presque rien face à ce que ses eaux ont signifié pour le chemin de la civilisation, de l'aube de l'histoire jusqu'à la conquête de l'Océan. Au présent, affirme le géographe, la Méditerranée a retrouvé sa position centrale sur les routes commerciales du monde, après l'ouverture du canal de Suez. L'une des preuves en est que la plus grande puissance maritime mondiale, la Grande-Bretagne, tient beaucoup à garder le contrôle des positions stratégiques de Gibraltar et Malte. Si l'Anglais ne contrôle pas à cemoment-là la sortie de Port-Saïd «il peut, s'il le veut, tirer le verrou à l'extrémité du long corridor extérieur que forme la mer Rouge, car ses garnisons veillent à l'îlot de Périm et sur le rocher d'Aden, à l'entrée de l'océan des Indes ${ }^{36}$. »

\section{L'Europe sur l'Océan}

Reclus est l'un des principaux géographes qui, entre les derniers décennies du XIX ${ }^{\mathrm{e}}$ siècle et les débuts du $\mathrm{XX}^{\mathrm{e}}$, appliquent la métaphore méditerranéenne aux océans pour éclairer

\footnotetext{
${ }^{32}$ Voir aussi : LEFORT I., «L'articulation littorale : un principe ritterien relu par Élisée Reclus », Études Rurales, n. 133,1994 , p. $45-58$.

${ }^{33}$ ReClus É., $N G U$, vol. I, op. cit., p. 47.

34 DEPREST F., «L'invention géographique de la Méditerranée : éléments de réflexion », L'Espace Géographique, n.1, 2002, p. 73-92.

${ }^{35}$ RECLUS É., $N G U$, vol. I, op. cit., p. 42.

${ }^{36}$ Ibid., p. 50.
} 
le phénomène explosif de la mondialisation, qui ne permettait plus de penser ni l'Europe ni la Méditerranée en tant que centre unique du monde. «L'analogie méditerranéenne relèverait d'une nouvelle façon de penser à l'échelle du monde, d'une perception non explicitée en tant que telle, voire confuse, de la mondialisation [...] Comparer les océans à la Méditerranée exprime le sentiment d'un glissement du centre du monde ${ }^{37}$. » Dans la NGU la métaphore méditerranéenne s'applique d'abord à l'Atlantique. Avec le progrès de la navigation à vapeur, les caractères de la petite mer intérieure sont transférés au grand océan. «L'océan Atlantique peut être considéré comme une "méditerranée". De même que la nappe "sans bornes" des eaux où se hasardaient avec terreur les premiers nautoniers hellènes finit par se révéler "mer close" [...] de même le formidable océan atlantique, encore tenu pour illimité il y a quatre siècles, se révèle à son tour comme une vallée sinueuse entre les deux moitiés de l'hémisphère continental $^{38}$.»

Reclus envisage, dans la partie de cet Océan qui se rattache le plus directement aux côtes européennes, une véritable «région liquide»: l'Atlantique Boréal. Cette aire correspond à la partie de l'Atlantique comprise entre le continent et la ligne idéelle qui va des Iles Britanniques à l'Islande, en se prolongeant à nord jusqu'aux îles Spitzberg. Pour le géographe, avoue Reclus, il est encore plus difficile d'envisager les limites des régions naturelles dans la mer que dans les continents. Là, les courants créent constamment des variations de température et salinité et les mesures bathymétriques donnent des résultats qui ne sont pas toujours fiables. «Beaucoup de problèmes restent à élucider dans l'étude de ces mouvements, car la connaissance des courants superficiels ne suffit pas ; il faut suivre aussi par la pensée tous les contre-courants cachés, observer la température changeante et salinité des eaux en toute leur épaisseur ${ }^{39}$. » Cependant, cette aire est bien définie par son uniformité climatique due au flux du courant de l'Atlantique tropical, qui réduit l'amplitude thermique saisonnière de ses eaux. «Sans lui les îles Britanniques et la Scandinavie seraient des pays inhabitables [...] il a donc une part capitale dans l'histoire moderne de l'humanitét ${ }^{40}$. »

Le mélange d'eaux venant de climats différents produit ici une richesse de faune maritime déterminante pour les activités humaines. La morue était poisson vital pour l'économie de plusieurs pays, ainsi que le hareng pour l'histoire de la Baltique et de la Frise : ses déplacements périodiques ont provoqué la fortune, ou bien la ruine, des communautés

\footnotetext{
37 ARRAUlt J.-B., «A propos du concept de méditerranée: expérience géographique du monde et mondialisation », Cybergeo, 2006, http://www.cybergeo.eu/index13093html.

${ }^{38}$ ReClus É., NGU, vol. XIV, Océan et Terres Océaniques, 1889, Paris, Hachette, p. 1.

${ }^{39}$ ReCLus É., $N G U$, vol. $I V$, op. cit., p. 4.

${ }^{40}$ Ibid., p. 14.
} 
riveraines de cette aire. Dans la métaphore reclusienne, les fonds de la mer du Nord et leurs pêcheries sont aussi productifs pour l'homme que «les vastes étendues de landes formant au sud une partie de son littoral ${ }^{41}$. » D'après le géographe certains fonds de cette mer, notamment le Dogger Bank, ont des caractères de continuité avec les plaines à demi-submergées de la Hollande et de la Frise. «La mer du Nord, étalant sa mince couche liquide sur le plateau qui porte les îles britanniques, offre ces excellents fonds de pêche précisément parce que elle est sans épaisseur et que son lit n'est couvert nulle part de pierres et de rochers ${ }^{42}$. »

Un fond doux, où la drague du pêcheur peut passer comme la charrue du paysan : la métaphore reclusienne relève d'une sorte d'équivalence topologique entre la plaine agricole et cette mer peu profonde où il suffit de jeter un filet pour s'approvisionner abondamment de nourriture. Cette équivalence est l'une des principales originalités de la démarche reclusienne : si la Méditerranée a été la mer des échanges et communications, la Mer du Nord a été le prolongement productif du continent européen, l'une des «plaines de la mer » ${ }^{43}$ qui ont favorisé la subsistance des populations septentrionales du continent.

C'est sur la mer qu'on a bâti, dans le sens littéral du mot, la nation hollandaise, qui a conquis centimètre par centimètre son territoire à l'eau et qui a toujours vécu en symbiose avec l'élément liquide : «Le sol de la basse Hollande, que les eaux de la mer ont tour à tour recouvert, a gardé, du moins en apparence, l'horizontalité d'une nappe liquide ${ }^{44}$. » L'ancien combat du peuple batave pour l'assèchement des polders n'est pas encore terminé, mais selon Reclus cette lutte avec la Mer du Nord est sous certains aspects une alliance. «L'Océan est tour à tour le protecteur et l'ennemi de la Hollande, Protector et hostis dit une ancienne médaille zélandaise : il la défend pendant la guerre, porte ses navires, apporte sur ses rivages des alluvions fécondes, mais il la menace aussi d'érosions constantes et d'inondations terribles $^{45}$. » Le géographe établit un lien entre les périodes où la conquête du terrain a été la plus importante et celles où la liberté républicaine donnait aux Provinces-Unies les institutions politiques les plus avancés de l'époque moderne. «L'œuvre de reconquête sur l'Océan ne prit une importance considérable qu'à la fin du seizième siècle, c'est-à-dire avec la période de la liberté républicaine. L'initiative individuelle, développée par la conscience de la grandeur nationale, fit entreprendre des travaux immenses, dont aucune autre contrée ne

\footnotetext{
${ }^{41}$ Ibid., p. 29.

${ }^{42}$ Ibid., p. 30.

${ }^{43}$ Ibid., p. 33.

${ }^{44}$ Ibid., p. 211.

${ }^{45}$ Ibid., p. 220.
} 
pouvait offrir d'exemples ${ }^{46}$. » Mais l'avantage le plus évident du littoral hollandais demeure sa fonction de véhicule de commerce, qui a favorisé les libertés citoyennes auxquelles Reclus donne beaucoup d'importance au fil de son œuvre.

Le géographe envisage une continuité entre cette expérience et une tradition datant du Moyen Age : «Chaque année les délégués des sept pays maritimes de la Frise se réunissaient prés d'Aurich, sur le territoire de la province actuelle d'Hanovre, et chacun des États se gouvernait lui-même [...] quel que fût le souverain étranger auquel la Frise appartînt nominalement, le peuple n'en restait pas moins le plus républicain d'Europe ${ }^{47}$. Reclus $^{\prime}$ remarque que de l'autre côté de la frontière, dans la Frise allemande, on garde encore le souvenir de cette histoire : «Les descendants des républicains du littoral gardent le souvenir de l'antique indépendance et répètent avec orgueil la devise de leur blason : Liewer dued üs Slav (Mort plutôt qu'esclave !) ${ }^{48}$.»

Reclus compare également la Ligue Hanséatique et ses emporiums de Brème, Lubeck, Bergen Danzig, aux villes «républicaines» italiennes, notamment Gênes et Venise, en confirmant ce lien entre navigation, commerce et villes libres.

La métaphore méditerranéenne est utilisée également à propos du littoral norvégien, développé par fjords, presqu'îles et îles. « On peut dire qu'il existe ainsi sur toute la côte de la Norvège une sorte de méditerranée, sinon pour l'étendue des eaux, du moins pour les routes maritimes, et c'est en effet dans le cordon des îlots extérieurs que se fait presque tout le mouvement du cabotage norvégien, dont l'importance est si considérable ; il n'est qu'un petit nombre de lieux où les embarcations soient obligées de se hasarder en pleine mer ${ }^{49}$. »

Reclus emploie un 'autre analogie méditerranéenne à propos de la navigation norvégienne de son époque : «Le pays des Phéniciens du Nord, étroit littoral contournant des monts et des plateaux inhabités, possède une marine plus puissante que de vastes pays ayant des dizaines de millions d'habitants comme la France, l'Espagne et la Russie ${ }^{50}$. »

\section{Conclusion}

Dans la NGU mer et montagne ont d'abord la fonction de définir et délimiter l'objet Europe. Ils jouent des rôles opposés sur le plan topologique : la montagne est la cuirasse du continent, tandis que la mer en est le véhicule des échanges; la montagne repousse ses fils

\footnotetext{
${ }^{46}$ Ibid., p. 245.

${ }^{47}$ Ibid., p. 235.

${ }^{48}$ RECLUS É., NGU, vol. III, op. cit., p. 739.

${ }^{49}$ ReClus É, NGU, vol. V, Europe Scandinave et Russe, Paris, Hachette, 1880, p. 78.

${ }^{50}$ Ibid., p. 161.
} 
vers les basses vallées par l'insuffisance de nourriture, tandis que la plaine liquide de la mer les soutient. La montagne représente l'histoire ancienne du continent, la mer son ouverture moderne et contemporaine.

Ces rôles ne sont toutefois jamais rigides et absolus : ils s'activent toujours à l'échelle de l'histoire, et leur opposition opère de façon réciproque et complémentaire, sur des temps que Fernand Braudel aurait nommés «géographiques ${ }^{51}$ ». Mer et montagnes s'opposent à la manière des pôles positif et négatif d'une pile électrique, métaphore que Reclus emprunte à Proudhon, mais qu'il réfère aussi à la Naturphilosophie de Schelling et Oken. L'hauteur et l'abîme sont aussi les deux directions algébriques d'une dimension physique, que la géographie européenne commence à intégrer entre le $\mathrm{XVIII}^{\mathrm{e}}$ et $\mathrm{XIX}^{\mathrm{e}}$ siècle, l'épaisseur du monde.

Enfin, l'aspect le plus original de la géographie d'un intellectuel hétérodoxe mais participant de la mentalité scientifique de son siècle est que mer, montagne et articulation littorale sont à l'origine de la puissance politique européenne. Reclus essaie toutefois de les insérer dans le cadre de sa proposition politique fédéraliste et libertaire.

\section{Sources imprimées}

Malte-Brun C., Précis de géographie universelle, Paris, F. Buisson, 1810-1829.

RECLus É., Nouvelle géographie universelle, Paris, Hachette, 1876-1894.

RECLus É., Histoire d'une montagne, Paris, Hetzel, 1880.

RITTER C., Géographie générale comparée, Bruxelles, Établissement Encyclographique, 1837.

RITTER C., Europa, Berlin, Georg Reimer, 1863.

RITTER C., Introduction à la géographie générale comparée, Paris, Les Belles Lettres, 1974.

Strabon, Géographie, Paris, Les Belles Lettres, 1969.

\section{Bibliographie}

ARRAUlt J.-B., «A propos du concept de méditerranée : expérience géographique du monde et mondialisation », Cybergeo, 2006, http://www.cybergeo.eu/index13093html.

BRAUdEL F., Écrits sur l'histoire, Paris, Flammarion, 1969.

BROC N., La géographie des philosophes : géographes et voyageurs français au XVIII siècle, Paris, Éditions Ophrys, 1974.

\footnotetext{
${ }^{51}$ BRAUDEL F., Écrits sur l'histoire, Paris, Flammarion, 1969, p. 11.
} 
BROC N., Les montagnes au siècle des Lumières, Paris, CTHS, 1991.

DEPREST F., «L'invention géographique de la Méditerranée: éléments de réflexion », L'Espace Géographique, n. 1, 2002, p. 73-92.

DunBar G., Élisée Reclus historian of nature, Amden, Archon Books, 1978.

FARINELli F., I segni del mondo, Firenze, la Nuova Italia, 1992.

FERRETTI F., Il mondo senza la mappa, Élisée Reclus e i geografi anarchici, Milano, Zero in Condotta, 2007.

LEFORT I., «L'articulation littorale : un principe ritterien relu par Élisée Reclus », Études Rurales, n. 133, 1994, p. 45-58.

Pelletier Ph., Élisée Reclus, géographie et anarchie, Paris, Éditions du Monde Libertaire, 2009.

RAFFESTIN C., «Storia di un ruscello », SCHMidT Di Friedberg M. (dir.), Élisée Reclus: natura e educazione, Milano, Bruno Mondadori, 2007, p. 294-296.

RoBIC M.-C. (dir.), Couvrir le monde, un grand $\mathrm{XX}^{e}$ siècle de géographie française, Paris, ADPF, 2006. 